\title{
Temozolomid über mehr als sechs Zyklen beim Glioblastom - sinnvoll oder nicht?
}

Hintergrund und Fragestellung: Für die kombinierte Radiochemotherapie des Glioblastoms sind sechs adjuvante Zyklen Erhaltungschemotherapie mit Temozolomid (TMZ) vorgesehen [1]. Oft werden jedoch deutlich mehr Zyklen eingesetzt. Retrospektive Analysen, die dieses Vorgehen unterstützen [2], sind dadurch limitiert, dass bei Patienten mit einem frühen Tumorprogress nie sechs oder mehr Zyklen erreicht werden. Gibt es einen Überlebensvorteil für Glioblastompatienten, bei denen die adjuvante TMZ-Erhaltungschemotherapie über mehr als sechs Zyklen fortgesetzt wird, oder nicht? Diese Frage wurde in einer aktuellen Studie geprüft [3].

Patienten und Methodik: Von 2.002 Patienten innerhalb des deutschen Gliomnetzwerks wurden 142 identifiziert, die mindestens sechs Zyklen einer adjuvanten TMZ-Erhaltungschemotherapie ohne Hinweise auf einen Tumorprogress erhiel-

Originalie

Gramatzki D et al. Limited role for extended maintenance temozolomide for newly diagnosed glioblastoma. Neurology. 2017;88(15):1422-30. ten. Bei 81 Patienten wurde nach sechs Zyklen die Chemotherapie beendet, bei 61 Patienten wurde sie im Median für insgesamt elf Zyklen fortgesetzt. Beide Gruppen wurden statistisch auf Vortei- le bzgl. des progressionsfreien (PFS) beziehungsweise Gesamtüberlebens (OS) mittels Log-rank-Test untersucht. Die Analyse von prognostischen Faktoren wie zum Beispiel dem Nachweis einer Mutation der Isozitratdehydrogenase (IDH) oder einer Promotor-Methylierung der O6-Methylguanin-DNA-Methyltransferase (MGMT) erfolgte uni- beziehungsweise multivariat mit der Cox-Regression.

Ergebnisse: Obwohl Patienten mit einer fortgesetzten TMZErhaltungschemotherapie über mindestens sieben Zyklen ein verlängertes PFS aufwiesen (20,5 vs. 17,2 Monate; $p=0,035)$, fand sich kein Vorteil beim OS (32,6 vs. 33,2 Monate; $p=0,126)$. Die multivariate Analyse - adjustiert für Alter, Ausmaß der Resektion, Karnofsky-Index, residuellen Tumor, MGMT-Promotor-Methylierung und IDH-Mutationsstatus - ergab keine Korrelation zwischen verlängerter TMZ-Erhaltungschemotherapie und PFS oder OS.

Schlussfolgerungen der Autoren: Diese Daten unterstützen nicht die Praxis, die TMZ-Erhaltungschemotherapie über sechs Zyklen hinaus fortzusetzen.

Schlüsselwörter: Glioblastom - Temozolomid - Therapiedauer

\section{- Kommentar von Martin Uhl, Erlangen}

\section{„Besser werden die Daten nicht"}

Die vorgestellte Arbeit der Züricher Arbeitsgruppe um Michael Weller ist anderen Untersuchungen überlegen, da in dieser Studie nur Patienten berücksichtigt wurden, die nach sechs adjuvanten TMZ-Zyklen noch keinen Hinweis auf einen Tumorprogress gezeigt hatten. Ältere retrospektive Studien hatten das Problem, dass die Gruppe mit weniger als sechs Zyklen auch Patienten mit einem frühen Tumorprogress enthielt, bei denen die TMZ-Erhaltungschemotherapie abgebrochen wurde. Bereinigt um diese Patienten ergab sich nun kein Vorteil der fortgesetzten Erhaltungschemotherapie mit TMZ. Vergleichbare Ergebnisse wurden kürzlich in einem anderen Datensatz von Blumenthal und Kollegen publiziert [4]. Der einzige Widerspruch zwischen beiden Studien war lediglich die Frage, ob nicht doch Patienten mit einem methylierten MGMT-Promotor von einer fortgesetzten TMZ-Erhaltungschemotherapie profitieren könnten. Dies bleibt aktuell noch ungeklärt.

Obgleich die hier vorgestellte Studie nicht die höchste Evidenzklasse hat, muss man leider feststellen, dass die Daten nicht besser werden. Um eine endgültige Klärung zu erreichen, bräuchte es eine prospektiv randomisierte Studie, in der nach sechs adjuvanten TMZ-Zyklen randomisiert wird, das heißt ca. neun bis zehn Monate nach der Operation. Angesichts der da-

für notwendigen enormen Patientenzahlen leuchtet es ein, dass es eine solche Studie niemals geben wird. Zusammenfassend kann man sagen, dass es - wenngleich die Anzahl der adjuvanten TMZ-Zyklen beliebig festgelegt wurde -, weiterhin keine Daten gibt, die ein Fortsetzen der TMZ-Erhaltungschemotherapie über sechs Zyklen hinaus unterstützen.
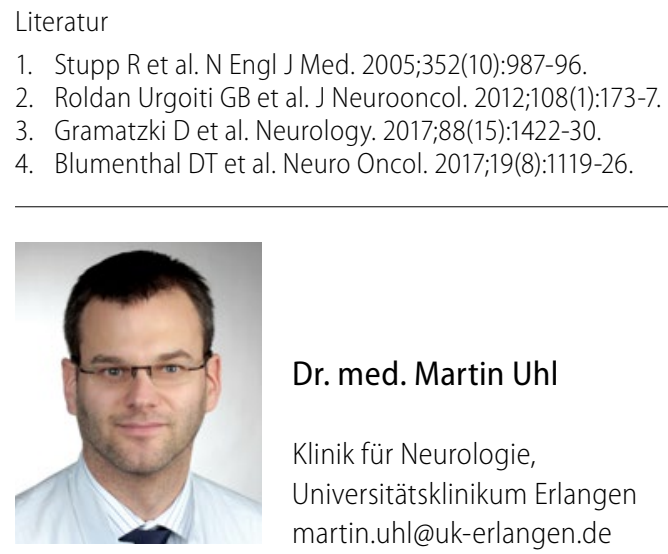

Dr. med. Martin Uhl

Klinik für Neurologie, Universitätsklinikum Erlangen martin.uhl@uk-erlangen.de 